Article

\title{
Analysis of Sensitivity, Linearity, Hysteresis, Responsiveness, and Fatigue of Textile Knit Stretch Sensors
}

\author{
An Liang ${ }^{1, *}\left(\mathbb{D}\right.$, Rebecca Stewart ${ }^{1,2}$ (D) and Nick Bryan-Kinns ${ }^{1}$ \\ 1 School of Electronic Engineering and Computer Science, Queen Mary University of London, \\ London E1 4NS, UK \\ 2 Dyson School of Design Engineering, Imperial College London, London SW7 2DB, UK \\ * Correspondence: a.liang@qmul.ac.uk; Tel.: +44-20-7882-7845
}

Received: 11 July 2019; Accepted: 13 August 2019; Published: 20 August 2019

\begin{abstract}
Wearable technology is widely used for collecting information about the human body and its movement by placing sensors on the body. This paper presents research into electronic textile strain sensors designed specifically for wearable applications which need to be lightweight, robust, and comfortable. In this paper, sixteen stretch sensors, each with different conductive stretch fabrics, are evaluated: EeonTex (Eeonyx Corporation), knitted silver-plated yarn, and knitted spun stainless steel yarn. The sensors' performance is tested using a tensile tester while monitoring their resistance with a microcontroller. Each sensor was analyzed for its sensitivity, linearity, hysteresis, responsiveness, and fatigue through a series of dynamic and static tests. The findings show that for wearable applications a subset of the silver-plated yarn sensors had better ranked performance in terms of sensitivity, linearity, and steady state. EeonTex was found to be the most responsive, and the stainless steel yarn performed the worst, which may be due to the characteristics of the knit samples under test.
\end{abstract}

Keywords: wearable; e-textiles; smart textiles; strain sensor; stretch sensor

\section{Introduction}

Wearable devices have been developed to track the human body in a number of different areas, such as fitness [1], healthcare [2], entertainment [3,4], and fashion [5]. However, in early wearable computer research, the sensors and computing system were put into a pocket to be carried on the body [6]. Since the 1970s and 1980s, clothing, accessories, and even the body itself were developed as an interface for various analog and digital functions by researchers [7]. The exploration of digital textile interfaces provides the opportunity for wearable technology to be integrated into our daily lives and could provide access to data generated by the body.

More recently, with an increasing range of applications for wearable devices research into intelligent textiles has attracted considerable attention [8-10]. Clothing is regarded as a second layer of skin for the human body - it keeps our body warm and protects us from the environment. On the basis of enhancing the basic functionality of clothing, researchers have started to explore its connectivity and interaction with surrounding objects and environment. "Smart clothing" [11], "wearables" [12], and "wearable computing" [6] are innovating what otherwise would be generic clothing [13]. Electronic textiles (e-textiles) combine textile technology and wearable computing to give fabric new functionality [14]. Combined with wearable computing, it can be an information processing system that has the ability to sense, feel, react, and act through wearer's subconscious or conscious actions and the surrounding environment [15]. Moreover, textiles provide enormous flexibility for 
system design with a wide range of fibers, yarn, fabrics, and manufacturing techniques can be developed to create products [15]. The development of textile sensors means wearable technology can move beyond handheld or pocket devices and be truly integrated into clothing [16-18].

One particular e-textile sensor that has attracted attention is the stretch or strain sensor. It has been used in an electronic sleeve for healthcare [19], in breathing monitoring applications [20], and to recognize upper body postures [21]. Stretch sensor properties are closely related to their fabrication and structure [22], and we are primarily interested in knit sensors that change their electrical resistance when under strain. For example, the number of wales (vertical knitted rows) and courses (horizontal knitted rows) of the conductive area, difference in structures like interlock or tubular knit, and also different wool blends and levels of stretch allowed by the fabric all feed into the sensor properties [23]. This follows on from earlier work by Metcalf et al. [24] who compared conductive yarn, knitting structures, and yarn compositions. They found that a single jersey knit structure with a silver-coated nylon yarn performed best for their sensor tracking knee movement. Later comparisons of knit sensors [25] found that the commercially produced Eeonyx fabric could be used to create more stable and less noisy sensors, but that other knitted fabrics may be preferable for applications of sensors that require a greater amount of stretch.

Whilst there are a wide range of applications for stretch sensors, our research is interested in using textile stretch sensors for dance applications, where the aesthetics and comfort of the sensors are highly valued alongside their sensing properties. There are several research areas where e-textiles may be used for dance applications, such as education [26] and new artistic expression [27]. Here, we examine how well the textile stretch sensors can detect movement with parameters similar to dance, focusing on materials that could be integrated at a later stage into a garment. Even though smart textiles sensors have been a subject of research for decades as outlined above, the literature has largely looked towards sports and medical applications. Textile sensors for dance movement and interactive art have not been examined extensively in a rigorous manner, though e-textiles have frequently been used in artistic works [28]. In this study, we evaluate the sensor properties of different conductive stretch fabrics in order to determine the most reliable sensor for dancer and computer interface design.

\section{Materials}

In this study, we compare twelve commercially produced conductive fabrics and four sensors knit in our research lab using two commercially available conductive yarns. These materials were chosen because they are commonly used by e-textile designers. The commercially produced knitted conductive fabrics have varying compositions, knit structures, and production methods which give the fabrics diverse properties. A full list all the sensors can be found in Table 1 with photos of each sensor in Figure 1. 
Table 1. Summary of samples.

\begin{tabular}{cccc}
\hline Sample No. & Composition & Knit Structure & Description \\
\hline 01 & Nylon 72\%/Elastan 28\% & single bed jersey knit & EeonTex \\
02 & Nylon 94\%/Elastomer 6\% & single bed jersey knit & Silver-plated \\
03 & Silver fiber 35\%/Cotton 40\%/Polyester 25\% & double bed interlock & Silver-plated \\
04 & Nylon 76\%/Elastic fiber 24\% & single bed jersey knit & Silver-plated \\
05 & Nylon 94\%/Elastomer 6\% & single bed jersey knit & Silver-plated \\
06 & Silver/Nylon 16\%/Rayon $84 \%$ & double bed interlock & Silver-plated \\
07 & silver-plated yarn & $1 \times 1$ rib & Silver-plated \\
08 & silver-plated yarn/Elastic yarn(100\%nylon) & $1 \times 1$ rib & Silver-plated \\
09 & Spun stainless steel yarn/Elastic yarn(100\%nylon) & $1 \times 1$ rib & Spun stainless steel \\
10 & Spun stainless steel yarn & $1 \times 1$ rib & Spun stainless steel \\
11 & Silver fiber/unknown & single bed jersey knit & Silver-plated \\
12 & Silver fiber 24.6\%/Cotton 36.8\%/Polyester 38.6\% & single bed jersey knit & Silver-plated \\
13 & Nylon Spandex & single bed jersey knit & Silver-plated \\
14 & $100 \%$ Silver fiber/Nylon spandex & single bed jersey knit & Silver-plated \\
15 & Polyamide 78\%/Elastomer 22\% & single bed jersey knit & Silver-plated \\
16 & $100 \%$ Silver fiber/unknown & single bed jersey knit & Silver-plated \\
\hline
\end{tabular}
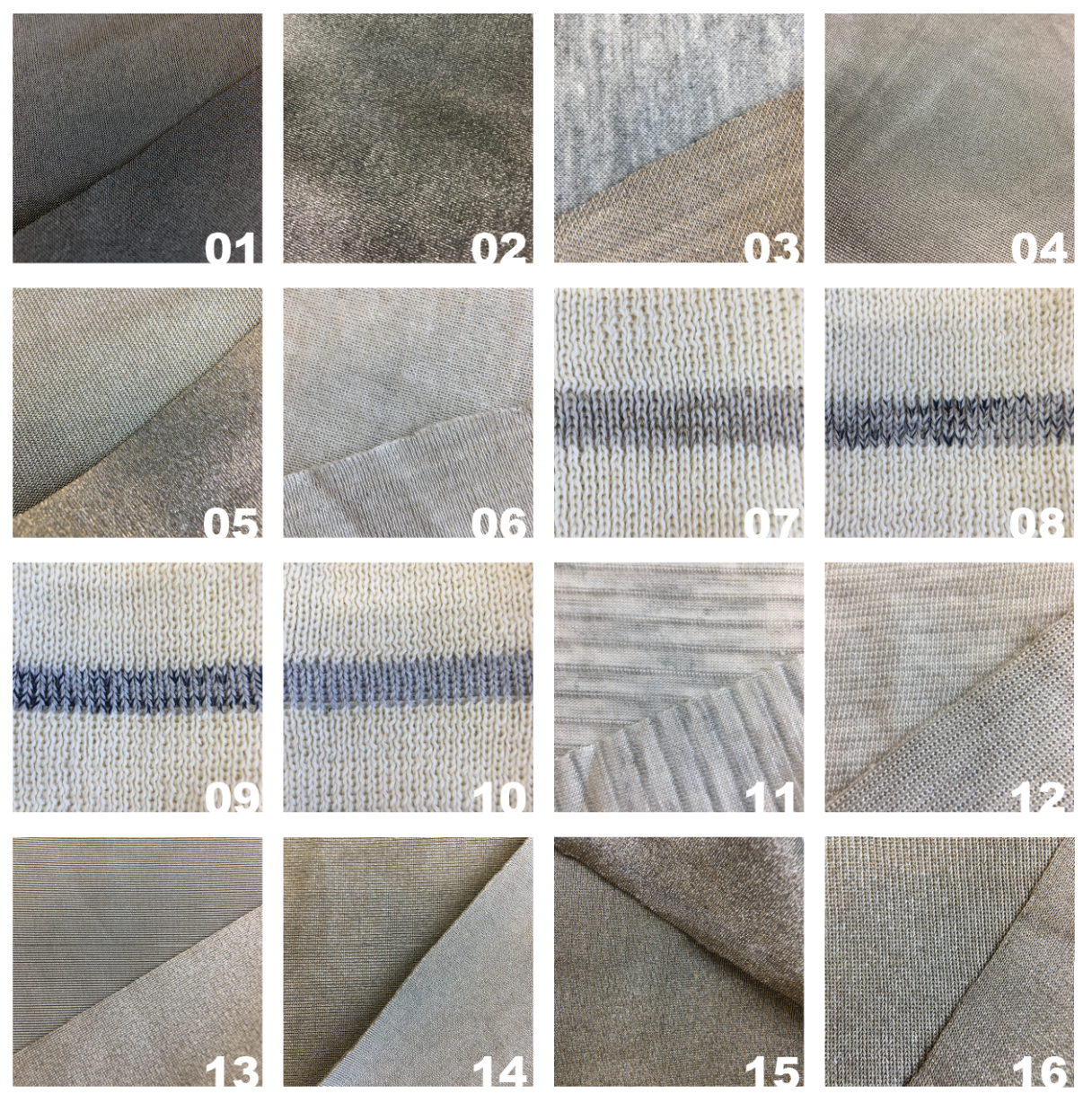

Figure 1. Photographs of the sixteen sensors along with their sensor number.

\subsection{Sensor Design}

Sixteen stretch sensors were constructed using the above materials. All the conductive fabrics were cut and the conductive yarns knit to the same dimensions of $20 \mathrm{~mm} \times 100 \mathrm{~mm}$ when under no strain. As Figure 2 shows, each piece of conductive fabric is stitched to a piece of woven fabric. The woven fabric is attached to the tensile testing machinery during the stretching tests. Each end of the conductive sensor fabric is electrically connected to a press-fit snap via conductive ripstop 
fabric. The snaps provide a wired connection to a breadboard containing a microcontroller resistance measuring circuit.

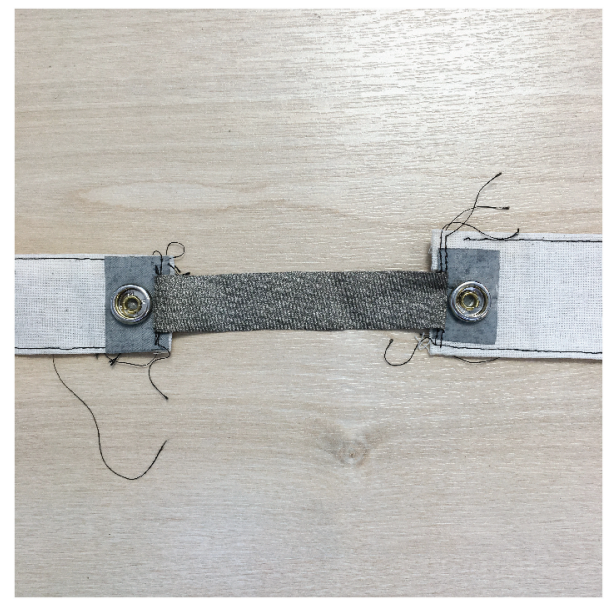

Figure 2. Sensor made with commercially produced conductive fabric.

The four sensors using commercially available conductive yarn (Samples 07 to 10) were knit in our research lab using a gauge 7 Dubied knitting machine (7 needles per inch) with a stitch tension of 9.5. The sensor is 6 rows in height, with a width of 30 needles on a double bed. All of the sensors start with 20 rows of white nonconductive wool (30/2 Nm Merino wool), then 6 rows of conductive yarn, then 20 rows of the white nonconductive wool again. Samples 08 and 09 are knit with blue elastane (Monofilament fiber: $100 \%$ Nylon, $70 / 23 \times 2$ ) throughout to enhance the elasticity and recovery. The same arrangement of conductive ripstop and snaps is used to connect the sensors to the microcontroller.

\subsection{Conductive Materials}

The sixteen stretch sensors each use one of three types of conductive materials: EeonTex; silver-plated yarn; and spun stainless steel yarn. Sample 01 is constructed from EeonTex, Samples 09 and 10 use spun stainless steel yarn, and all other samples are silver-plated yarn knitted sensors from commercially available fabrics or threads. Samples 07 to 10 are knit with a $1 \times 1$ rib knitting structure, Samples 03 and 06 have a double bed interlock knitting structure, all other sensors are knit with single bed jersey knit. Samples 07 to 10 were knit in our research lab using an industrial Dubied knitting machine to compare silver-plated and spun stainless steel yarns.

\subsubsection{EeonTex}

Sample 01 is made from EeonTex LTT-SLPA, a conductive stretchable fabric produced by Eeonyx Corp. This material consists of $72 \%$ nylon and $28 \%$ spandex elastic fabric coated with a proprietary conductive polymer. It has a two-way stretch (warp and weft) and can be used for pressure and stretch sensor applications. This material is washable, but after 30 cycles of washing, there is no longer a significant change in resistance when the fabric is stretched or pressed [29].

\subsubsection{Spun Stainless Steel Yarn}

The stainless steel sensors, Sample 09 and 10, were knit in our research lab using a spun stainless steel yarn $(10 / 3 \mathrm{Nm})$ from Plug \& Wear Srl. The spun yarn is $20 \%$ stainless steel and $80 \%$ polyester and consists of three threads plied together. The weight of the yarn is 10 meters per gram, resulting in a yarn count of $10 / 3 \mathrm{Nm}$. 


\subsubsection{Silver-Plated Yarn}

Silver is a popular material for conductive textiles as it has a low electrical resistance and is safe to be worn against the skin. It is most commonly plated around a nonconductive fiber which is then plied and woven or knit into a textile. There are many kinds of silver-plated materials in the market and Samples 02 to 08 and Samples 11 to 16 are silver-plated sensors with different material compositions and knit structures. Sample 02 uses MedTex 180, Sample 05 uses MedTex P-180, and Sample 15 uses Technik-tex P130B, all from Statex Produktions. Samples 11 to 14 and Sample 16 are from Chinese shielding fabrics manufacturer (DaZhi, QingDao, China). Sample 11 uses silver fiber antibacterial conductive cloth (DaZhi \#3-17), Sample 12 uses metal fiber radiation protection fabric (DaZhi \#3-2), Sample 13 uses $135 \mathrm{~g} \mathrm{100 \%} \mathrm{silver} \mathrm{fiber} \mathrm{four-way} \mathrm{stretch} \mathrm{fabric} \mathrm{(DaZhi} \mathrm{\# 3-18),} \mathrm{Sample} 14$ uses $130 \mathrm{~g}$ 100\% silver fiber four-ways stretch fabric (DaZhi \#3-15), Sample 16 uses 100\% silver fiber ion cloth (DaZhi \#3-8-1). Samples 03, 04, and 06 are from Less EMF Inc. (Ithaca, NY, USA), with corresponding product numbers Cat.\#1281, \#A321, and \#1224. In addition, Shieldex 110/34 dtex, 2-ply yarn from Statex Produktions is knit in Samples 07 and 08.

\section{Methods}

To evaluate the performance of the sensors for use in dance applications, we use two types of evaluation approaches to cover a range of movements that may occur in dance performance: (i) Evaluation of the sensors held in a static steady-state; and (ii) dynamic repeated movements of stretching and relaxing the sensors. An Instron 5900 Series Universal Testing Instrument (Instron, Wycombe, UK) was used to stretch the sensors.

To determine the maximum extension being measured, we recorded the maximum stretched length of the elbow joint bending on three people with different heights and genders ( 2 females, $1.60 \mathrm{~m}$; and 1 male, $1.80 \mathrm{~m}$ ). We found that the stretching range of the elbow joint was similar across our small sample-all around $60 \mathrm{~mm}$. Accordingly, the extension value of tensile testing has been set as $60 \mathrm{~mm}$.

\subsection{Resistance Calculation}

As the sensors under test all exhibit a piezoresistive effect, the resistance is the key parameter to observe. Therefore, we use a highly accurate measurement method to calculate the resistance changes of these sensors-the Wheatstone bridge circuit.

The Wheatstone bridge [30] is an electrical circuit that can accurately measure resistance. There are four resistors connected to the two legs of the bridge. Given the three known resistor values, we can calculate the unknown resistor's value by balancing two legs of the bridge.

$$
R_{X}=\frac{\frac{R_{3}}{R_{1}+R_{3}}+\frac{V_{m}}{V_{b}}}{1-\frac{R_{3}}{R_{1}+R_{3}}+\frac{V_{m}}{V_{b}}} R_{2} .
$$

In this study, we set up a Wheatstone bridge circuit for every sample. There are three known resistances, $R_{1}, R_{2}$, and $R_{3}$, with $R x$ being the stretch sensor. The value of $R_{x}$ is calculated by Equation (1), using an Arduino Uno microcontroller to read the voltage from the two sides of the bridge $\left(V_{1}\right.$ and $\left.V_{2}\right)$, then by calculating $V_{m}$. The 5 volt supply voltage from the Arduino board is denoted at $V_{b}$. The values of $R_{1}, R_{2}$, and $R_{3}$ are dependent on the resistance of the sensor $\left(R_{x}\right)$, so we measured the resistance of each sensor when stretched and released using a multimeter. The values for $R_{1}, R_{2}$, and $R_{3}$ are the mean of these two resistance values, rounded to the closest value of a commercially available resistor.

\subsection{Dynamic Test}

The samples are tested starting at $10 \%$ strain to ensure they are always under some tension. In the dynamic test, samples are stretched and released 100 times, from $110 \mathrm{~mm}$ to $170 \mathrm{~mm}$, at a 
speed of $6 \mathrm{~mm} / \mathrm{s}$. The aim of this test is to evaluate the reliability of the sensors by analyzing the relationship between strain and the electrical resistance of the sensor, and whether that relationship remains consistent over multiple cycles. When looking towards dance applications, this will determine the extent to which the sensor could reliably detect body movement, such as the angular change of a joint like an elbow.

The tensile tester stretched the sensor and recorded the strain and extension exerted, also, in parallel, a separate microcontroller measured the electrical resistance. A sampling rate of $128 \mathrm{~Hz}$ was chosen as a sufficiently high rate as $120 \mathrm{~Hz}$ is the upper limit of motion capture cameras.

\subsection{Static Test}

The samples are initially stretched at a speed of $6 \mathrm{~mm} / \mathrm{s}$, then stopped and held at $70 \%$ strain for three minutes. Then, the samples are released at $6 \mathrm{~mm} / \mathrm{s}$ and held for three minutes at $10 \%$ strain. This test examines how stable the sensor is over time, especially as the textile settles.

\subsection{Data Analysis}

The following metrics are extracted using the above data capture methods.

\subsubsection{Working Range and Gauge Factor}

The working range of the sensors is the maximum and minimum electrical resistance values at the minimum and maximum stretch. Here, we measure each sensors working range as the maximum and minimum electrical resistance values of the sensor stretched from $10 \mathrm{~mm}$ to $70 \mathrm{~mm}$.

Gauge factor (GF) is defined as the ratio of relative changes in electrical resistance $(\Delta R)$ to the mechanical strain $(\varepsilon)$, described in the following equation:

$$
\mathrm{GF}=\frac{\Delta R / R}{\Delta L / L}=\frac{\Delta R / R}{\varepsilon},
$$

where $R$ is the initial resistance, $\Delta R$ is the change in resistance, $L$ is the initial length, and $\Delta L$ is the change in length.

\subsubsection{Linearity}

In this study, we look at the linear relationship between resistance and strain during stretching and relaxation in the dynamic test, where one hundred cycles of stretching and relaxation data are aggregated and the start and end of linear regions identified. In an ideal sensor, the resistance change should have a linear relationship to the extension so that the extension can be inferred from the resistance measurement.

The best-fit line for the aggregated stretch and relaxation data is found and the root-mean-square error (RMSE) for that line is calculated. The sensor with the least error can be assumed to be the most accurate for measuring movement. Figure 3 illustrates the identification of the linear region and the fit line. 


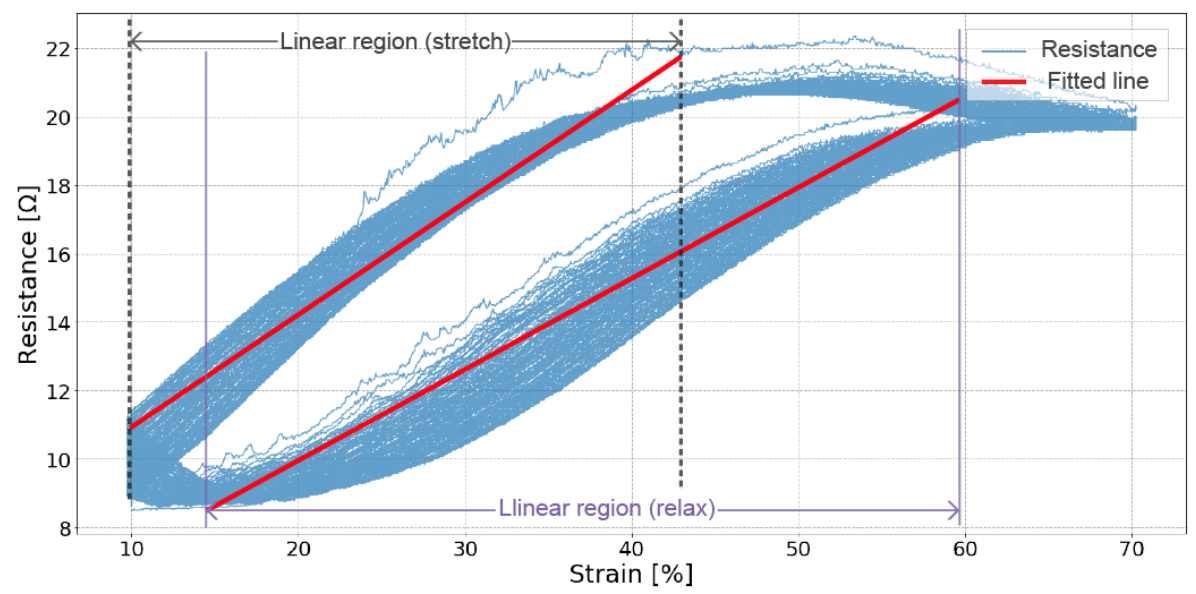

Figure 3. An example of linearity fitting of the dynamic test for Sample 04. The linear region of stretch and relaxation are identified and then fit with a line.

\subsubsection{Hysteresis}

The dynamic test looks at the relationship between resistance and strain over repeated measures. The electrical resistance performs differently when the sensor is stretched compared to when it is relaxed, and the maximum difference between stretch and relax is the hysteresis. Figure 4 illustrates an example of the measured hysteresis. It is calculated by fitting a third order curve to the stretch and relaxation data, then calculating the maximum difference of the two curves.

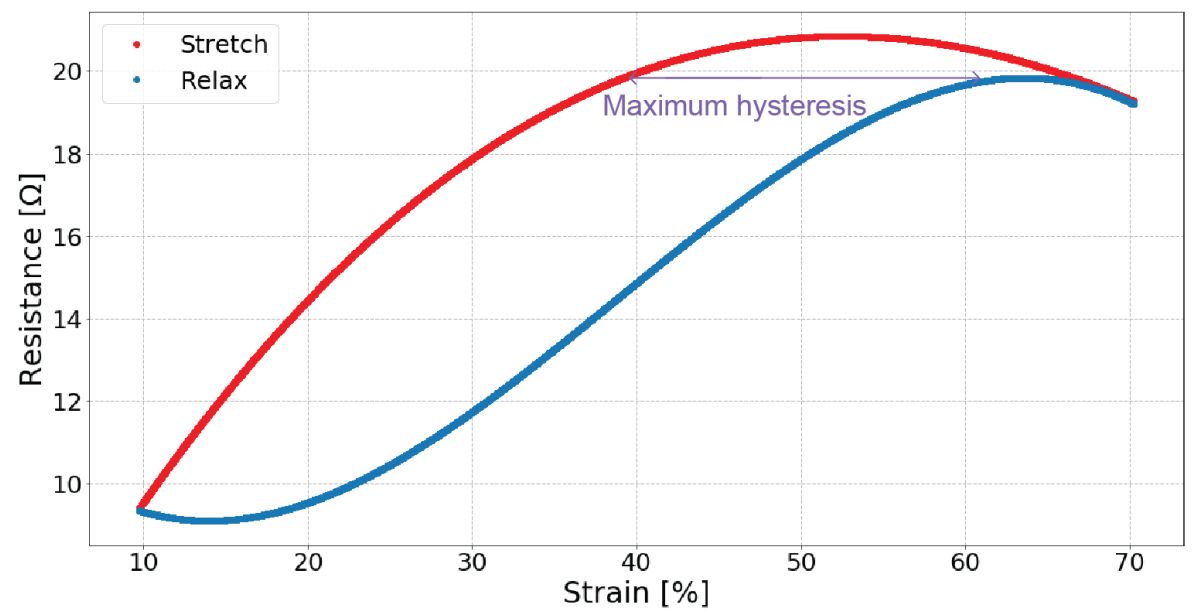

Figure 4. An example of the maximum hysteresis in the aggregated data from the dynamic test (Sample 04).

\subsubsection{Fatigue}

In the dynamic test, we examine the repeatability of the sensors over one hundred cycles, and fit the linear region of stretch and relaxation of the aggregate cycle data, then calculate the error of each stretch and relaxation cycle from that fit line. The difference of each cycle's error compared with the sensor's average error is examined to determine when fatigue first occurs.

\subsubsection{Responsiveness}

Responsiveness is defined as how fast the sensor responds electrically to a change in physical direction. We measure the response time when changing from stretching to relaxing and vice versa. We determine the nonlinear region by visual observation, then measure the length of time of the nonlinear region in dynamic and static tests. In the dynamic test, we measured the average nonlinear 
region of one hundred cycles in two directions, from relax to stretch and from stretch to relax. In the static test, we measure the nonlinear region when samples are initially held at $70 \%$ strain (stretched) and 10 strain\% (relaxed), as shown in Figure 5.

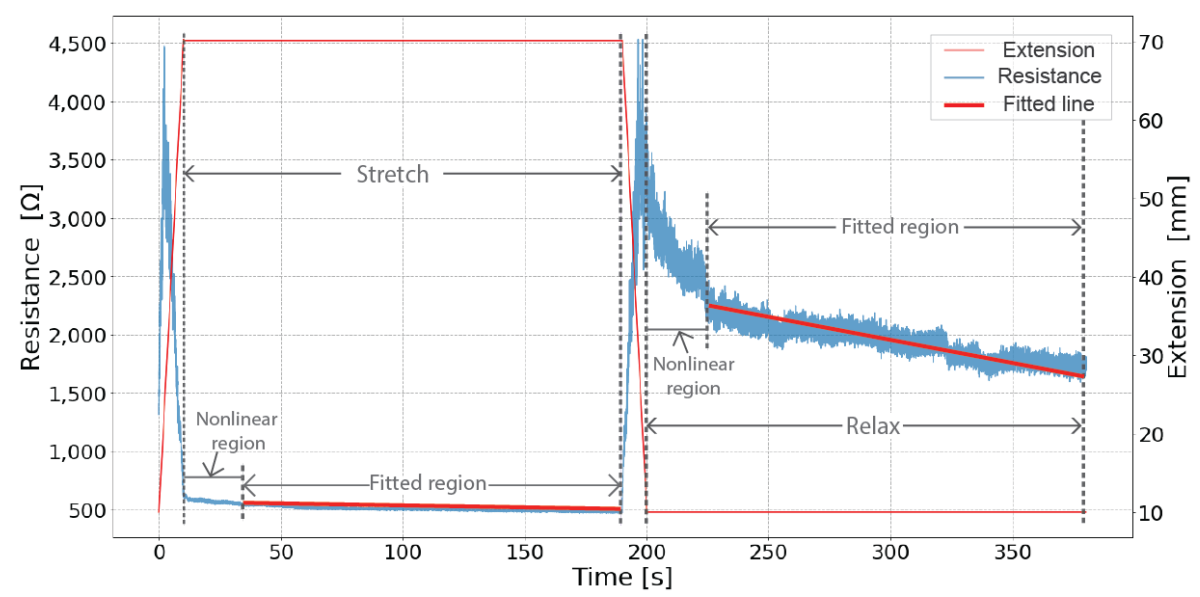

Figure 5. An example of linearity fitting a static test with Sample 9. The linear region is fit with a line when the sample is held in a stretched and relaxed position.

\subsubsection{Steady State}

The static test investigates each sensor's performance when under constant strain. As Figure 5 shows, two parts of the static test data are examined: When the sensor is stretched to $70 \%$ strain and held for three minutes; and when relaxed to $10 \%$ strain and held for three minutes. For most of the sensors, there is a nonlinear region when the sensors' state is first changed. The time until the linear region begins again is noted, then a line is fit to the subsequent region. The RMSE and the slope of the line are compared.

\section{Results}

\subsection{Working Range and Gauge Factor}

The working range of the sensors is the resistance range produced from under no strain, the unstretched length of $100 \mathrm{~mm}$, to a stretched length of $170 \mathrm{~mm}$. The initial electrical resistance of the sensors is measured using Rapid 318DMM Digital Multimeter before the subsequent tests to avoid fatigue influence the results. Table 2 shows the gauge factor as calculated from the sensor's working range and resistance when under no strain. 
Table 2. Working range and gauge factor of sensors.

\begin{tabular}{cccc}
\hline Sample No. & Initial Resistance $(\Omega)$ & Working Range $(\Omega)$ & Gauge Factor \\
\hline 01 & 55,857 & 40,715 & 1.215 \\
02 & 727.62 & 1297.6 & 2.972 \\
03 & 8.36 & 4.34 & 0.866 \\
04 & 12.92 & 20.22 & 2.608 \\
05 & $13.87 \mathrm{M}$ & $12.29 \mathrm{M}$ & 1.477 \\
06 & 20.86 & 16.43 & 1.313 \\
07 & 667.48 & 902.92 & 2.255 \\
08 & 565.11 & 1309.77 & 2.318 \\
09 & 225.30 & 3706.36 & 27.418 \\
10 & 771.43 & 6822.09 & 14.739 \\
11 & $14.92 \mathrm{M}$ & $15.79 \mathrm{M}$ & 1.763 \\
12 & $352.82 \mathrm{~K}$ & $489.68 \mathrm{~K}$ & 2.313 \\
13 & 39.80 & 24.29 & 1.018 \\
14 & 8.40 & 56.82 & 11.273 \\
15 & 62.73 & 56.40 & 1.498 \\
16 & 4.25 & 3.40 & 1.333 \\
\hline
\end{tabular}

Silver-plated sensors of Samples 05, 11, and 12 have relatively high initial electrical resistance values. Sample 05 had a large electrical resistance and produced such a noisy signal that we could not get valuable data from this sensor during the tests, therefore this sensor's data is not analyzed in the following sections.

Spun stainless steel knitted sensors (Sample 09, 10) and one silver-plated sensor (Sample 14) have much larger gauge factors than the other sensors, almost over ten times higher than all the other sensors.

\subsection{Linearity}

We did not analyze the dynamic test data of the two sensors knit in our lab with conductive yarn, Samples 09 and 10. Their resistances became very large, passing almost no electrical current, after the completion of the dynamic test. This is possibly because they became deformed and lost their elasticity after being stretched one hundred times.

Table 3 summarizes the linearity performance of each sensor during stretching and Table 4 during relaxing. We identified each sensor's linear region, the average resistance of the sensor while stretching, and the RMSE. Each sensor's RMSE was divided by their average resistance $(\bar{R})$ to normalize the results.

Figures 6-8 respectively show the result of Sample 15, Sample 1 and Sample 8. Each shows the output from one hundred stretches and identifies the linear region fit with a line. They show examples of a silver fabric, EeonTex, and a lab-knitted sensor and are also illustrative examples of the best performing (Figure 6), middle performing (Figure 7), and the worst performing (Figure 8) results. 


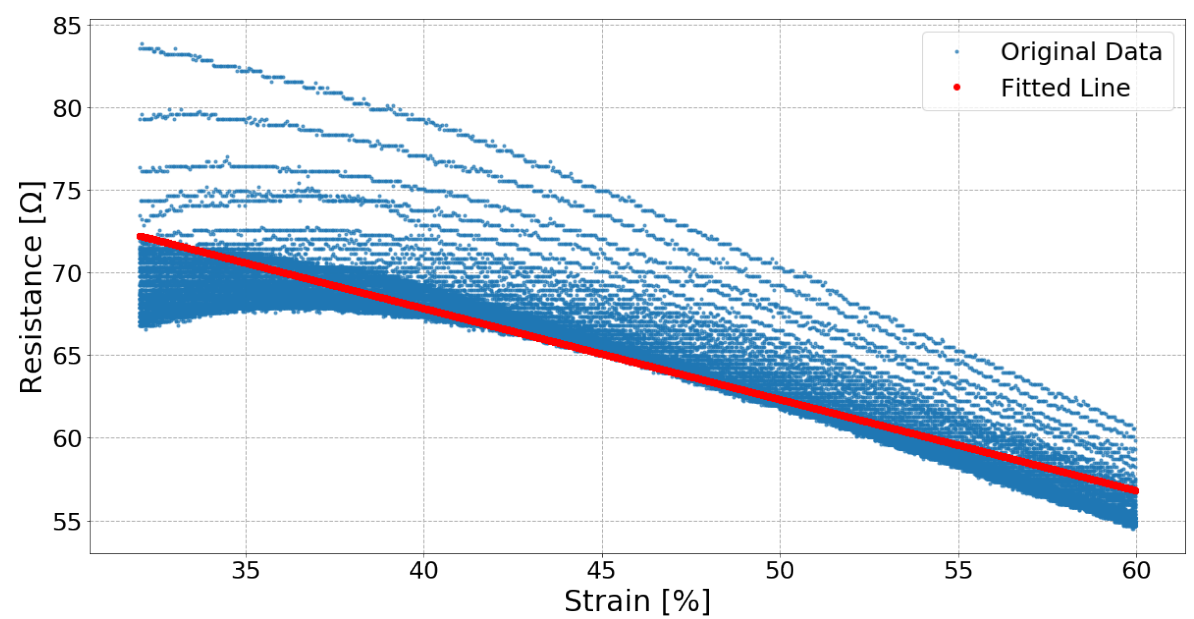

Figure 6. Sample 15, a silver-plated sensor (Technik-tex P130B), response and the fitted line from 32\% strain to $60 \%$ strain while stretching.

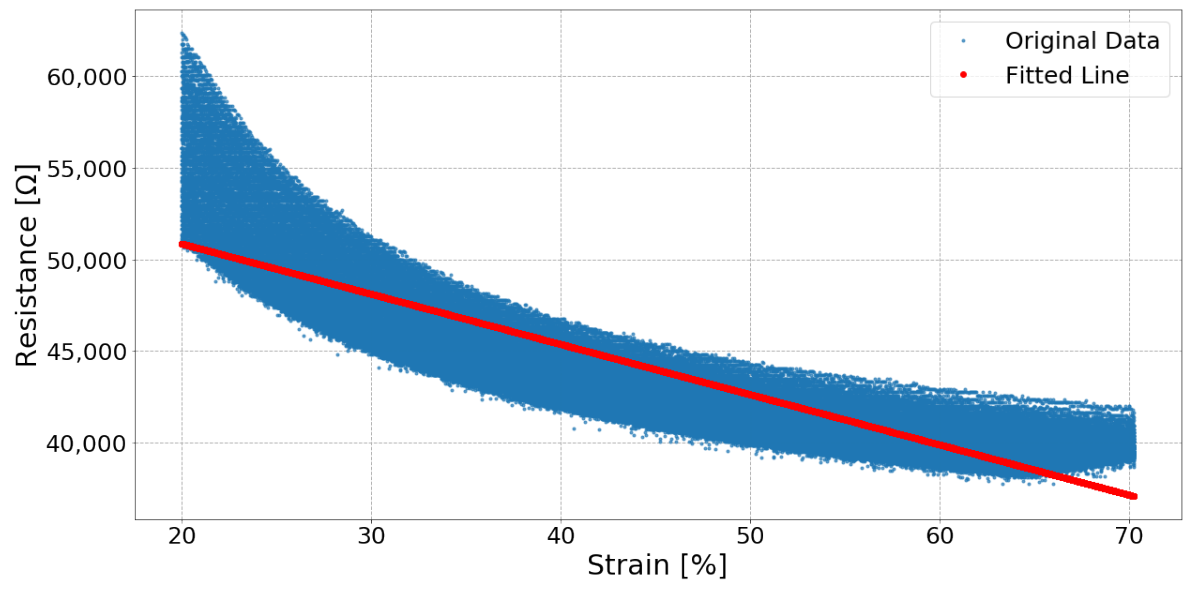

Figure 7. Sample 01 (EeonTex) sensor response and the fitted line while stretching between $20 \%$ strain and $70 \%$ strain.

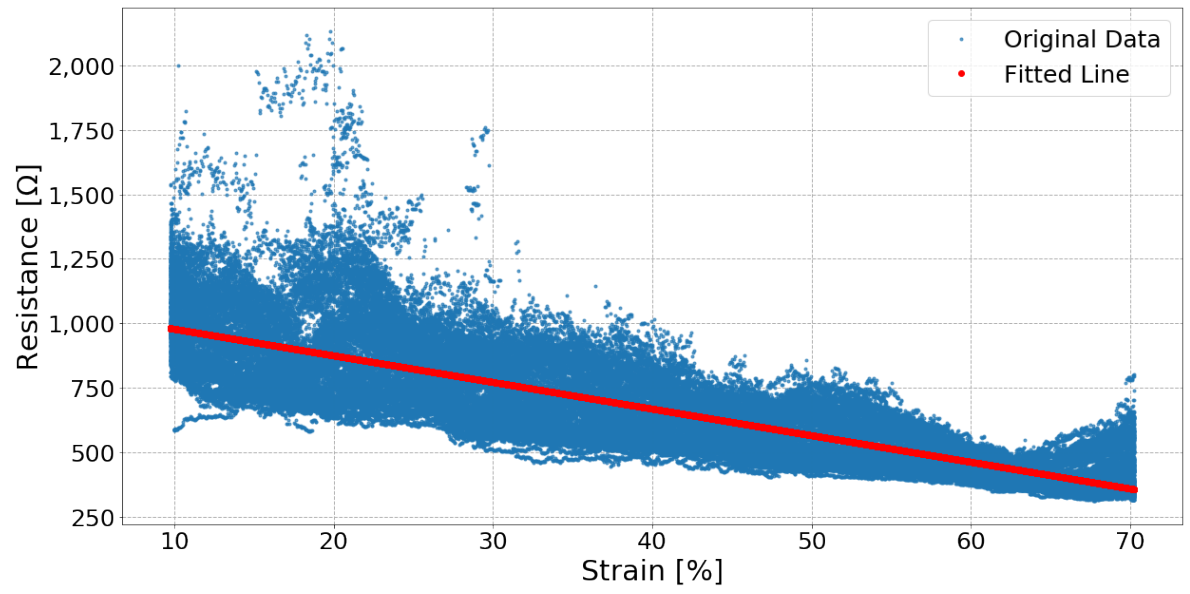

Figure 8. Sample 08, a lab-knitted sensor, response and fitted line of one hundred cycles of stretching between $10 \%$ strain and $70 \%$ strain. 
Table 3. Each sample's linearity performance while stretching (ranked by root-mean-square error (RMSE)). Samples 05, 09, and 10 did not produce usable data, so were excluded from the analysis.

\begin{tabular}{cccc}
\hline Sample No. & Linear Region $($ Strain/\%) & Stretching $\bar{R}(\Omega)$ & RMSE $/ \bar{R}$ \\
\hline 15 & $32-60$ & 62.401 & 0.0280 \\
03 & $18-70$ & 7.399 & 0.0337 \\
16 & $20-59$ & 4.833 & 0.0382 \\
13 & $23-70$ & 38.777 & 0.0394 \\
01 & $20-70$ & $43,942.283$ & 0.0479 \\
04 & $10-42$ & 16.377 & 0.0496 \\
06 & $18-58$ & 16.773 & 0.0626 \\
02 & $30-70$ & 284.615 & 0.1061 \\
11 & $10-70$ & $15,555,332.216$ & 0.1084 \\
14 & $28-70$ & 9.827 & 0.1316 \\
07 & $10-70$ & 533.365 & 0.1933 \\
08 & $10-70$ & 669.435 & 0.1977 \\
12 & $10-70$ & $803,969.899$ & 0.9825 \\
05 & - & - & - \\
09 & - & - & - \\
10 & - & - & - \\
\hline
\end{tabular}

Table 4. Each sample's linearity performance while relaxing (ranked by RMSE). Samples 05, 09, and 10 did not produce usable data, so were excluded from the analysis.

\begin{tabular}{cccc}
\hline Sample No. & Linear Region (Strain/\%) & Relaxing $\bar{R}(\boldsymbol{\Omega})$ & RMSE/ $\bar{R}$ \\
\hline 13 & $36-70$ & 37.609 & 0.0353 \\
01 & $20-70$ & $45,676.049$ & 0.0368 \\
03 & $20-70$ & 8.080 & 0.0437 \\
16 & $10-60$ & 5.396 & 0.0463 \\
15 & $41-70$ & 62.763 & 0.0479 \\
14 & $28-70$ & 9.048 & 0.0512 \\
04 & $14.7-57$ & 14.367 & 0.0544 \\
06 & $14.6-70$ & 18.517 & 0.0599 \\
11 & $10-70$ & $16,945,000.200$ & 0.1239 \\
02 & $30-70$ & 419.864 & 0.1909 \\
07 & $10-70$ & 807.640 & 0.3041 \\
08 & $10-70$ & 995.857 & 0.3201 \\
12 & $10-70$ & $1,407,711.161$ & 0.9507 \\
05 & - & - & - \\
09 & - & - & - \\
10 & - & - & - \\
\hline
\end{tabular}

\subsection{Hysteresis}

The maximum measured hysteresis for each sensor is shown in Table 5. Figure 4 can be referred to as an example plot of the hysteresis.

Table 5. Maximum hysteresis error of the sensors.

\begin{tabular}{cc}
\hline Sample No. & Maximum Hysteresis (Approx.) \\
\hline 01 & $7 \%$ \\
03 & $13 \%$ \\
11 & $15 \%$ \\
12 & $15 \%$ \\
02 & $16 \%$ \\
15 & $17 \%$ \\
14 & $21 \%$ \\
04 & $24 \%$ \\
13 & $25 \%$ \\
\hline
\end{tabular}


Table 5. Cont.

\begin{tabular}{cc}
\hline Sample No. & Maximum Hysteresis (Approx.) \\
\hline 16 & $27 \%$ \\
07 & $28 \%$ \\
06 & $29 \%$ \\
08 & $32 \%$ \\
05 & - \\
09 & - \\
10 & - \\
\hline
\end{tabular}

\subsection{Fatigue}

During one hundred cycles of stretching and relaxation, 9 of the sensors show resistance change and 10 of the sensors show signs of fatigue. With the increasing cycles, resistance of Sample 1 decreases and resistance of Samples 2, 3, 6, 7, 8, 11, 12, and 16 increases. Table 6 shows the number of cycles when sensors were fatigued as indicated by a change from decreasing to increasing error (RMSE).

Figures 9-11 respectively showed examples of each silver-plated fabric, EeonTex, and lab-knitted sensor's response of one hundred stretch linear region and their fitted line, they are also the illustrative examples of the best (Figure 9), average (Figure 10), and worst (Figure 11) results.

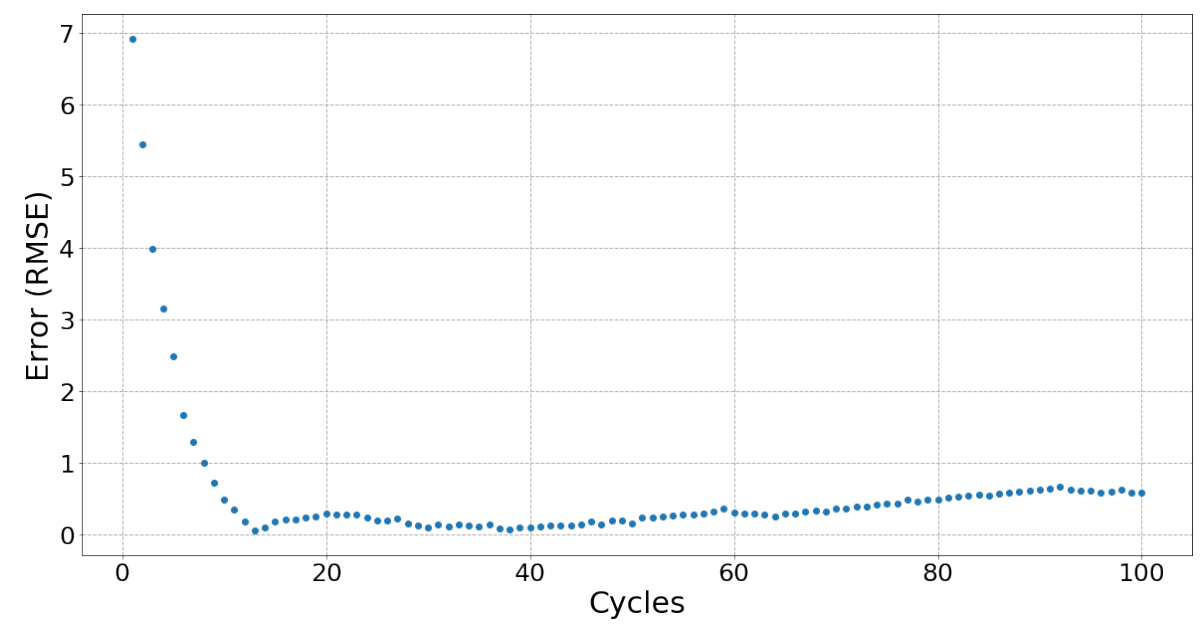

Figure 9. The error between each cycle of stretch and the fitted line of Sample 15. This shows the best repeatability result from all the samples' stretch and relax.

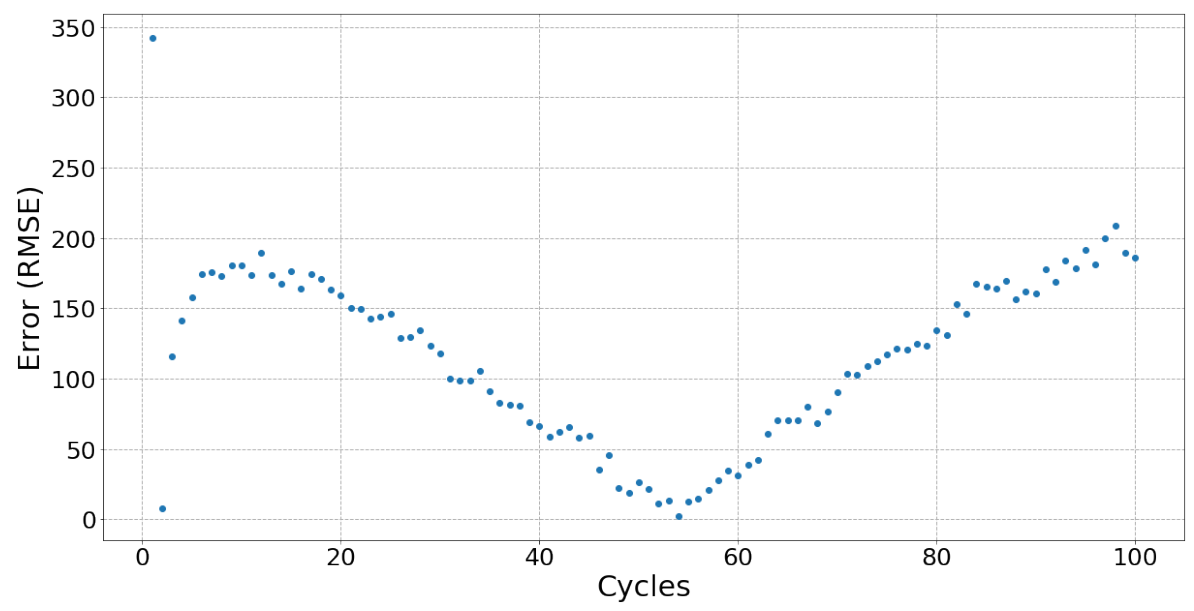

Figure 10. The error between each cycle of relaxation and the fitted line of Sample 01 (EeonTex). This plot shows the typical repeatability result from all the samples' stretch and relax. Most of the samples showed fatigue after 50 cycles of stretching. 


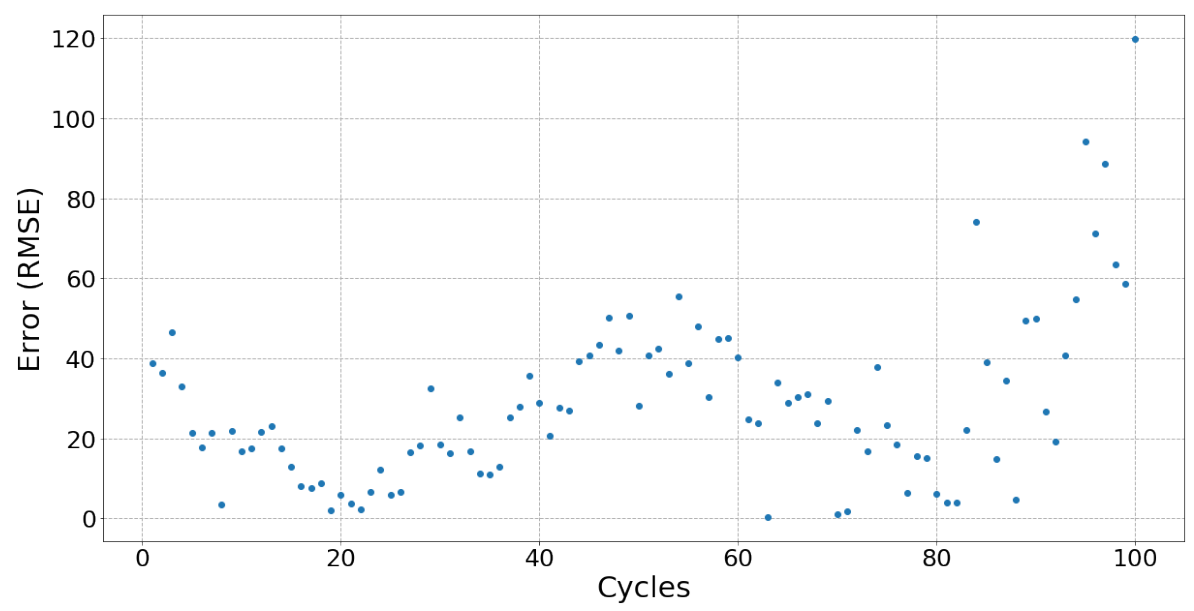

Figure 11. The error between each cycle of stretching and the fitted line of Sample 07. This plot shows a relatively worse repeatability result from all the samples' stretch and relax.

Table 6. The cycle number at which sensors showed signs of fatigue.

\begin{tabular}{ccc}
\hline Sample No. & Stretch (Cycles) & Relaxation (Cycles) \\
\hline 01 & 82 & 52 \\
02 & 80 & 77 \\
03 & 0 & 0 \\
04 & 77 & 77 \\
05 & - & - \\
06 & 79 & 73 \\
07 & 81 & 58 \\
08 & 72 & 77 \\
09 & - & - \\
10 & - & - \\
11 & 88 & 89 \\
12 & 79 & 74 \\
13 & 79 & 90 \\
14 & 94 & 71 \\
15 & 0 & 75 \\
16 & 0 & 0 \\
\hline
\end{tabular}

\subsection{Responsiveness}

In order to measure how quickly the sensors can respond to a change of movement direction, we calculate the time elapsed between a stopping or reversing of movement and when the sensor's resistance returns to a linear output. Table 7 shows the response times when stopping at both $70 \%$ strain (sensors being stretched) and 10\% strain (sensors being relaxed), and the sensor's response time in dynamic tests when reversing from two direction-relaxing to stretching and stretching to relaxing. Figure 12 illustrates each of the response times.

Table 7. Sensors' response time.

\begin{tabular}{ccccc}
\hline Sample No. & At $\mathbf{7 0} \%$ Strain (s) & At $\mathbf{1 0} \%$ Strain (s) & Relax to Stretch (s) & Stretch to Relax (s) \\
\hline 01 & 3.936 & 1.919 & 0.667 & 0 \\
02 & 4.568 & 13.989 & 1.333 & 0 \\
03 & 2.304 & $180+$ & 1.417 & 0 \\
04 & 16.102 & 19.389 & 0 & 1.500 \\
05 & - & - & - & - \\
06 & 10.125 & 12.430 & 1.583 & 0 \\
07 & 11.185 & 20.039 & 1.500 & 0 \\
08 & 3.413 & 8.45 & 2.000 & 0.833 \\
\hline
\end{tabular}


Table 7. Cont.

\begin{tabular}{ccccc}
\hline Sample No. & At $\mathbf{7 0} \%$ Strain (s) & At $\mathbf{1 0} \%$ Strain (s) & Relax to Stretch (s) & Stretch to Relax (s) \\
\hline 09 & 12.787 & 24.26 & - & - \\
10 & 7.484 & 14.400 & - & - \\
11 & 1.17 & 8.959 & 0 & 3.667 \\
12 & 9.067 & 8.939 & 1.750 & 1.083 \\
13 & 10.504 & 5.5 & 2.333 & 0.500 \\
14 & 4.328 & 11.269 & 1.700 & 0 \\
15 & 13.494 & 7.449 & 0 & 0 \\
16 & 13.066 & $180+$ & 2.417 & 0.800 \\
\hline
\end{tabular}
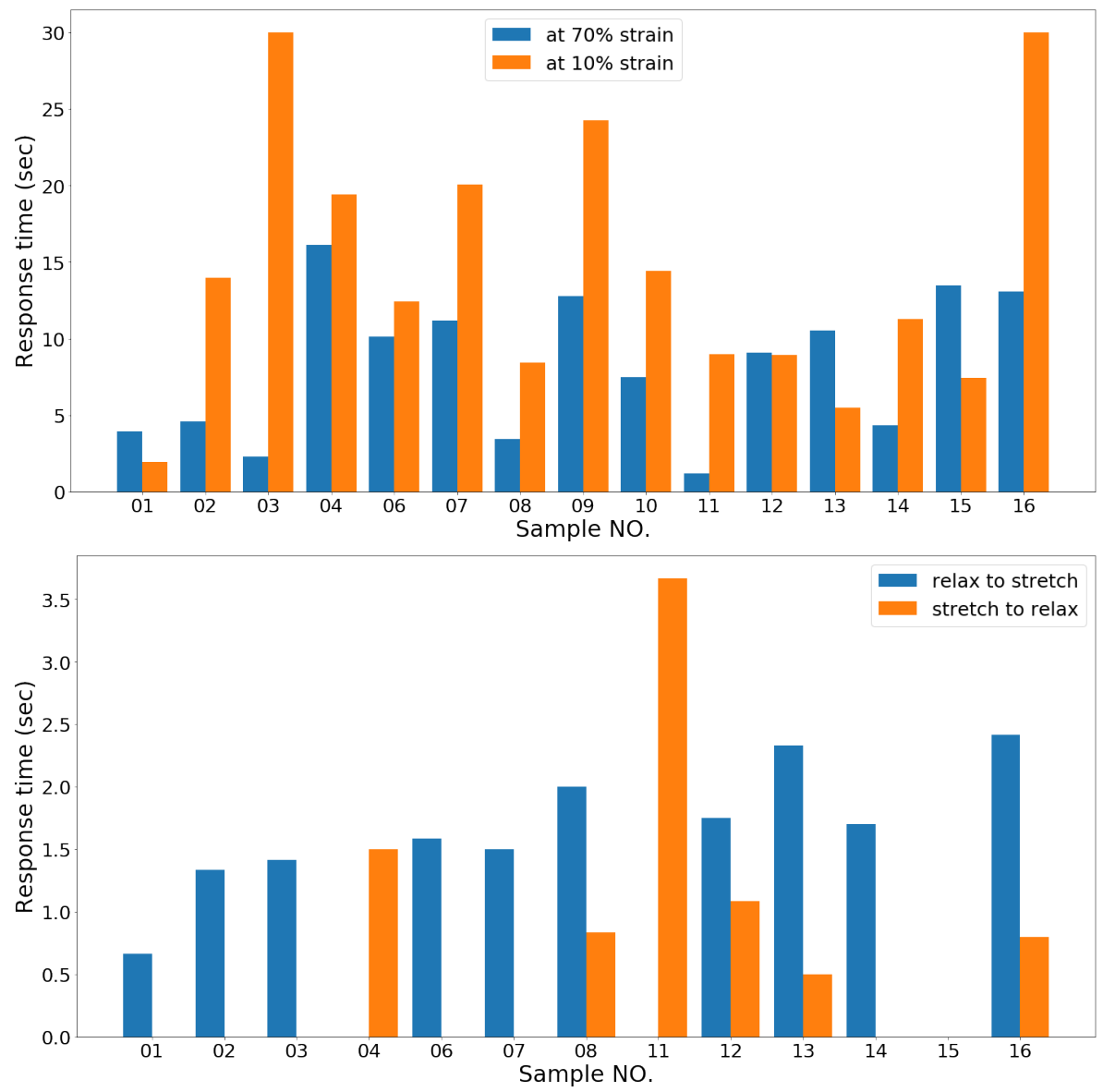

Figure 12. Comparison of each sensor's response time in static (top) and dynamic (bottom) tests.

\subsection{Steady State}

Table 8 shows each sensor's information when they are held at a constant strain of $70 \%$, the elapsed time of the nonlinear region, the average resistance value when the sensor is stretched, the RMSE of the fitted line, and the slope of the fitted line. The nonlinear time is how long the nonlinear region lasts at the beginning after the sensor is stretched and first held at $70 \%$ strain. As the sensors have different scales of resistance, their averaged resistance is calculated and used to normalize the RMSE for each sensor.

Figure 13 shows an example of the line fitting, which is the average result of sensors held at a constant strain of $70 \%$, and Table 9 shows each sensor' analysis when held at a constant strain of $10 \%$. Samples 16 and 03 are too noisy to fit a meaningful line. 
Table 8. Summary of sensors held at 70\% strain (ranked by slope).

\begin{tabular}{cccc}
\hline Sample No. & Stretched $\overline{\boldsymbol{R}}$ & RMSE/ $\overline{\boldsymbol{R}}$ & Fitted Line Slope \\
\hline 14 & 7.380 & 0.0024 & 0.00000178 \\
16 & 3.404 & 0.0039 & -0.00000354 \\
13 & 26.437 & 0.0019 & -0.00001151 \\
04 & 19.539 & 0.0029 & -0.00001853 \\
15 & 48.376 & 0.0021 & -0.00002187 \\
07 & 264.348 & 0.0067 & -0.00040201 \\
12 & 239.792 & 0.0831 & -0.00088288 \\
03 & 6.169 & 0.0032 & -0.00093813 \\
09 & 500.327 & 0.0134 & -0.00243984 \\
10 & 749.237 & 0.0117 & -0.00277595 \\
06 & 13.715 & 0.0029 & -0.00454466 \\
02 & 108.481 & 0.0027 & -0.00808992 \\
08 & 307.380 & 0.0325 & -0.03222228 \\
01 & $53,311.627$ & 0.0034 & -0.03268672 \\
11 & $6,851,052.653$ & 0.0104 & -15.50951161 \\
05 & - & - & - \\
\hline
\end{tabular}

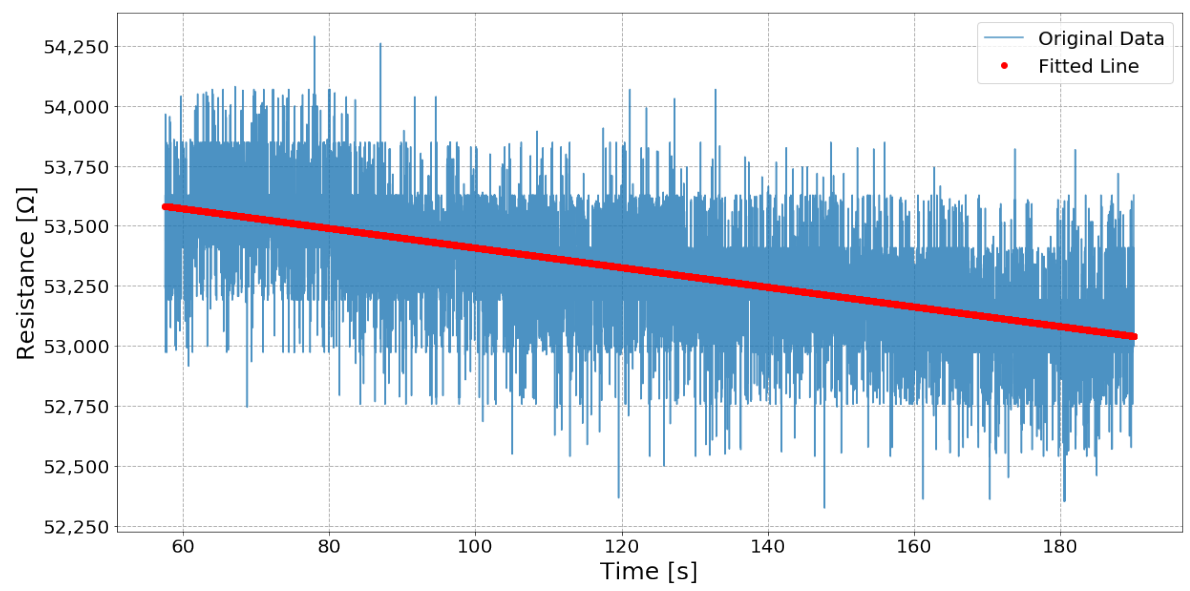

Figure 13. The fitting of Sample 1 while the sensor is at 70\% strain. The EeonTex is a representational average result of all the samples.

Table 9. Results of sensors held at a constant strain of 10\% (ranked by slope).

\begin{tabular}{cccc}
\hline Sample No. & Relaxed $\overline{\boldsymbol{R}}$ & RMSE/ $\overline{\boldsymbol{R}}$ & Fitted Line Slope \\
\hline 16 & 4.736 & 0.0375 & -0.00000571 \\
03 & 8.690 & 0.0162 & 0.00000706 \\
04 & 9.307 & 0.0028 & 0.00001066 \\
15 & 32.456 & 0.0022 & -0.00001234 \\
13 & 24.834 & 0.0021 & -0.00003154 \\
14 & 8.431 & 0.0069 & -0.00003731 \\
06 & 16.689 & 0.0037 & -0.00004653 \\
07 & 665.198 & 0.0142 & -0.00019367 \\
02 & 452.813 & 0.0040 & -0.00144364 \\
08 & 616.229 & 0.0111 & -0.00178679 \\
10 & 2239.344 & 0.0118 & -0.01216064 \\
09 & 1933.900 & 0.0274 & -0.02570112 \\
01 & $102,725.338$ & 0.0080 & -0.33534839 \\
12 & $104,871.145$ & 0.0139 & -1.24070950 \\
11 & $16,616,606.761$ & 0.0143 & -28.49458985 \\
05 & - & - & - \\
\hline
\end{tabular}




\section{Discussion}

\subsection{Working Range and Gauge Factor}

The working range and gauge factors are important starting points when considering a sensor for a given application, such as capturing the movement of a dancer's body. Sensors with larger gauge factors are more suitable for a variety of measurement locations on the body, especially along a joint where large angular movements need to be tracked. However, sensors with a small gauge factor may still be useful for movements where less fine-grained measurements are sufficient. When considering only the gauge factor, the two sensors with stainless steel yarn, Samples 09 and 10 appear to perform the best, but this is the only metric in which they perform well. They have a large working range, likely because of the structure of the spun conductive yarn which exhibits piezoresistivity even before being placed in the knit structure. With the exception of Sample 14, whose exact materials are unknown, all the silver-plated sensors have a similar gauge factor ranging from 0.87 to 2.97 . The EeonTex, Sample 01, falls roughly in the middle of that range at 1.22. However, the gauge factor alone gives very limited insight into the performance of a sensor-further analysis is needed.

\subsection{Linearity}

An ideal sensor has a linear relationship between the input and output-in this case, the extension of the sensor ideally has a linear relationship with the electrical resistance of the sensor. This was evaluated by identifying what region of the sensor output could reasonably be considered linear, then fitting a line to that region and calculating the resulting error. The linear region was identified by manually inspecting the aggregated 100 cycles of stretching the sensor and 100 cycles of relaxing the sensor.

There is not a clearly identifiable sensor with the most ideal linearity performance as no single sensor had the largest linear region and also the least error when being stretched and relaxed. However, Samples 01, 03, and 15 all performed well in aggregate across these metrics. For example, Sample 15 was found to have the least error compared to its fit line when being stretched, but its linear region is only from $32 \%$ strain to $60 \%$ strain, which is a smaller range than most of the other sensors. Sample 03 has a slightly larger error than Sample 15 when stretched, but a much larger linear region from $18 \%$ to $70 \%$ strain. Therefore, when using the sensors to track movement, Sample 15 would predict the movement more precisely but for a limited range of motion. Sample 03 would track almost the entire range of a movement up to $70 \%$ strain, but with less precision.

Two of the lab-knitted sensors, Samples 09 and 10, were discarded during these tests as their resistances became so large as to approximate not passing any electrical signal. This is likely related to the sensors becoming significantly deformed and losing their elasticity after one hundred stretching cycles. The other pair of lab-knitted sensors with silver-plated yarn, Samples 07 and 08, also produced noisy signals probably due to their relatively loose knit structure.

Generally, the sensors' RMSE measurements are larger when relaxing than when stretching. We propose this is because, during stretching, the sensor's resistance changes with an external force consistently being applied, so the sensor's performance is relatively stable. However, when being relaxed, there is no longer an external force being applied, so the performance of the sensors is fully dependent on the material's ability to rebound. Therefore, the relaxation also reflects the elasticity of the sensor's material and structure.

Measuring the hysteresis during stretching and relaxation quantifies the differences between the two movement directions, with an ideal sensor containing no hysteresis. Sample 01, EeonTex, has the smallest amount of hysteresis with the silver-plated sensors having diverse values ranging from $13 \%$ to $29 \%$. The more-elastic sensors tend to have the lowest hysteresis. The lab-knitted sensors have the largest hysteresis of around 30\%, indicating that the lab-knitted sensors take longer to recover. They are knit at a significantly larger gauge than the commercially manufactured fabrics, which may also play a role. 


\subsection{Fatigue}

When used in applications such as tracking body movement, sensors are stretched more than once, meaning that fatigue may affect the performances of a sensor over time. Only two sensors, Samples 03 and 16, did not show signs of fatigue after 100 cycles of stretching and relaxing, with Sample 15 showing no signs of fatigue during stretching but did after 75 relaxation cycles. Figure 9 shows Sample 15 with no fatigue during stretching as the error is kept consistent, unlike Figure 10, which shows a trend typical for most of the sensors with errors increasing after 75 cycles. For most sensors, there appears to be a "breaking in" period (around 10 to 20 cycles) when there is greater error in the beginning of the cycles which then gradually stabilizes. The exceptions are the lab-knitted sensors, seen in Figure 11, which are less stable overall until about 80 cycles when fatigue sets in.

\subsection{Responsiveness}

The ideal performance of a stretch sensor has an identical linear performance during both stretching and relaxation and can instantaneously transition between these two states. This is particularly important in body movement when tracking, for example, a joint like an elbow opening and closing. In reality, this is not physically possible to do absolutely instantaneously due to the material constraints, but not all materials have the same responsiveness.

In the static test, no sensor has a linear performance instantly when the sensor stops moving at both $70 \%$ strain and 10\% strain, but each sensor has a different response depending on whether the sensor stopped after being stretched or released. As Table 7 shows, in the static test, most of the sensors take less time return to linear performance when it has been stretched and held at $70 \%$ strain, likely because there is an external force stretching the sensors and keeping them stable. Among all the samples, Samples 01, 03, and 11 were ranked with the quickest response times when held at $70 \%$ strain. However, Samples 01, 13, and 15 have quicker responses when they are stopped after relaxation than after stretching, which indicates these three samples have good elasticity which rebound when the sensors have been released. As Figure 12 shows, Sample 01, EeonTex, is the most responsive in general in the static test.

In the dynamic test, the sensors have quicker responses than in the static test as the sensors are kept constantly moving, but some of the sensors still have nonlinear regions when their movement reverses. The results show that the sensors respond more quickly when transitioning from a stretching state to a relaxing state. We propose this is because after several cycles of stretching and relaxation, most of the sensors are deformed, causing an increase in the length of the sensors, so the sensors are not under tension at the beginning of the cycle. Figure 12 shows Sample 04, 11, and 15 have effectively instantaneous response times when the samples change direction from relaxing to stretching. This indicates that these sensors have good elasticity and are not deformed after several cycles of stretching. When examining the change from stretching to relaxing, most of the sensors have effectively instant response times. Sample 15, a silver-plated sensor, has $0 \mathrm{~s}$ response times in both directions of the dynamic test so it is the best sensor for dynamic motion tracking.

\subsection{Steady State}

When held under steady tension, an ideal sensor outputs a static value. However, a textile sensor changes and settles over time with several factors influencing this behavior.

A line fit to the linear region of a sensor under constant strain ideally has a slope of 0 . When held at $70 \%$ strain, Sample 14 performed the best under that metric, but was slightly noisier than Sample 13 which had the third best slope. Sample 13 was also the least noisy under a constant $10 \%$ strain, though Sample 04 had the best slope under that condition.

We found the error and fitted line slope is larger when the sensors are relaxed, likely because the sensors have no external force being applied and instead rely entirely on their own elastic rebound during the relaxation. The more-elastic sensors have a more stable performance, such as Sample 15 
which has the best result when relaxed. It has slightly larger resistance and RMSE than Sample 13 but shows better fitted line slope, which means Sample 15 has better elasticity and stability when relaxed.

Combining the results of sensors held at $70 \%$ and $10 \%$ strain, we found the sensors which have small average resistances are less noisy and also have better a slope. Samples 13 and 15 performed better in the static test in general as they are less noisy and have the smallest slope. Sample 01, the EeonTex, has a larger error and slope, especially when stretched. Surprisingly, it performed worse than all the lab-knitted sensors.

The steady state of the sensors would reflect how well the sensors will present the pause in the middle of dance movements. For example, when a dancer bends their elbow and keeps the position for a while, then changes to another position. The sensors which have small resistance and better elasticity will be able to sense these kinds of movements in real time.

\section{Conclusions}

This study compared sixteen stretch sensors constructed from commercially available materials, evaluating metrics that best align with the desired characteristics of wearable sensors for tracking body movement. We concentrated on different aspects of the linearity of the sensor responses and how they respond in dynamic motions, looking at sensitivity, hysteresis, responsiveness, and fatigue. We found the sensitivity or gauge factor of a given sensor was a poor predictor of how it would perform in subsequent tests. What is more important is the choice of materials and structure, with commercially knit silver-plated yarn at a tight gauge yielding the most consistent success.

Overall, we found Samples 13 and 15 to have the best general results, especially in respect to linearity. Both of the samples are silver-plated yarn, single bed jersey knits. They have relatively small working range, so they would be good to use for small-scale body movement tracking such as in the muscle, back, and spine. EeonTex has the least hysteresis and fastest responsiveness, so it is particularly suited to rapid, dynamic movement tracking. When compared to the commercially produced fabrics, the lab-knitted sensors did not work well, especially the stainless steel sensors. Among the four lab-knitted sensors, the samples knitted with silver yarn performed better than the stainless steel samples, though the addition of elasticated yarn had mixed results. The addition of elastic only improved the responsiveness and not the other metrics. However, all of the lab-knitted sensors had significantly looser gauges that likely contributed to their poor performance. With the identification of materials and structures that do perform better, future work can encompass recreating those sensor characteristics directly into knit garments.

This study only examined a small selection of characteristics under controlled settings. Before these sensors can be robustly used in real-world applications, future work around the effect of sweat and body heat along with considerations regarding washing, color, texture, and maintenance is needed.

Author Contributions: Conceptualization, A.L.; Data curation, A.L.; Formal analysis, A.L.; Investigation, A.L.; Methodology, A.L. and R.S.; Supervision, R.S. and N.B.K.; Validation, R.S.; Visualization, A.L.; Writing-original draft, A.L.; Writing—review and editing, R.S. and N.B.K.

Funding: This work was supported by the EPSRC and AHRC Centre for Doctoral Training in Media and Arts Technology (EP/L01632X/1) and China Scholarship Council.

Acknowledgments: The authors would like to thank Sophie Skach for preparing the lab-knitted sensors, Giulio Moro for his support of the early stage of data analysis, and Kening Wan for her help with equipment set up.

Conflicts of Interest: The authors declare no conflict of interest.

\section{References}

1. Mauriello, M.; Gubbels, M.; Froehlich, J.E. Social fabric fitness: The design and evaluation of wearable E-textile displays to support group running. In Proceedings of the SIGCHI Conference on Human Factors in Computing Systems, Toronto, ON, Canada, 26 April-1 May 2014; pp. 2833-2842. 
2. Byrne, C.A.; Rebola, C.B.; Zeagler, C. Design research methods to understand user needs for an etextile knee sleeve. In Proceedings of the 31st ACM International Conference on Design of Communication, Greenville, NC, USA, 30 September-1 October 2013; pp. 17-22.

3. Chu, N.N.; Yang, C.M.; Wu, C.C. Game interface using digital textile sensors, accelerometer and gyroscope. IEEE Trans. Consum. Electron. 2012, 58, 184-189. [CrossRef]

4. Vega-Barbas, M.; Pau, I.; Ferreira, J.; Lebis, E.; Seoane, F. Utilizing smart textiles-enabled sensorized toy and playful interactions for assessment of psychomotor development on children. J. Sens. 2015, 2015. [CrossRef]

5. Seymour, S. Fashionable Technology: The Intersection of Design, Fashion, Science, and Technology; Springer: New York, NY, USA, 2008.

6. McCann, J.; Bryson, D. Smart Clothes and Wearable Technology; Elsevier: Amsterdam, The Netherlands, 2009.

7. Ryan, S.E. Garments of Paradise: Wearable Discourse in the Digital Age; MIT Press: Cambridge, MA, USA, 2014.

8. Boczkowska, A.; Leonowicz, M. Intelligent materials for intelligent textiles. Fibres Text. East. Eur. 2006, 59, 13-17.

9. Norstebo, C.A. Intelligent textiles, soft products. J. Future Mater. 2003, 1-14.

10. Tomico, O.; Wilde, D. Soft, embodied, situated \& connected: Enriching interactions with soft wearables. mUX J. Mob. User Exp. 2016, 5, 3.

11. Van Langenhove, L.; Hertleer, C. Smart clothing: A new life. Int. J. Cloth. Sci. Technol. 2004, 16, 63-72. [CrossRef]

12. Chan, M.; EstèVe, D.; Fourniols, J.Y.; Escriba, C.; Campo, E. Smart wearable systems: Current status and future challenges. Artif. Intell. Med. 2012, 56, 137-156. [CrossRef] [PubMed]

13. Lee, S.; Du Preez, W.; Thornton-Jones, N. Fashioning the Future: Tomorrow's Wardrobe; Thames and Hudson: London, UK, 2005.

14. Barnes, N. Smart Textiles for Designers-Inventing the Future of Fabrics. J. Text. Des. Res. Pract. 2016, 4, 191-194. [CrossRef]

15. Schneegass, S.; Amft, O. Smart Textiles: Fundamentals, Design, and Interaction; Springer: Cham, Switzerland, 2017.

16. Harjuniemi, E.; Colley, A.; Rytilahti, P.; Li, H.; Forest, J.; Häkkilä, J. Idle stripes shirt: Ambient wearable display for activity tracking. In Proceedings of the 2018 ACM International Symposium on Wearable Computers, Singapore, 8-12 October 2018; pp. 254-259.

17. Brueckner, S.; Freire, R. Embodisuit: A wearable platform for embodied knowledge. In Proceedings of the 2018 ACM International Symposium on Wearable Computers, Singapore, 8-12 October 2018; pp. 242-247.

18. Lévai, Z.; Setét, S. Power of proximity metacommunication between garments. In Proceedings of the 2018 ACM International Symposium on Wearable Computers, Singapore, 8-12 October 2018; pp. 278-283.

19. Yang, K.; Meadmore, K.; Freeman, C.; Grabham, N.; Hughes, A.M.; Wei, Y.; Torah, R.; Glanc-Gostkiewicz, M.; Beeby, S.; Tudor, J. Development of user-friendly wearable electronic textiles for healthcare applications. Sensors 2018, 18, 2410. [CrossRef] [PubMed]

20. Guo, L.; Peterson, J.; Qureshi, W.; Kalantar Mehrjerdi, A.; Skrifvars, M.; Berglin, L. Knitted Wearable Stretch Sensor for Breathing Monitoring Application; Ambience'11: Borås, Sweden, 2011.

21. Mattmann, C.; Amft, O.; Harms, H.; Troster, G.; Clemens, F. Recognizing upper body postures using textile strain sensors. In Proceedings of the 2007 11th IEEE International Symposium on Wearable Computers, Boston, MA, USA, 11-13 October 2007; pp. 29-36.

22. Seyedin, S.; Razal, J.M.; Innis, P.C.; Jeiranikhameneh, A.; Beirne, S.; Wallace, G.G. Knitted strain sensor textiles of highly conductive all-polymeric fibers. ACS Appl. Mater. Interfaces 2015, 7, 21150-21158. [CrossRef] [PubMed]

23. Atalay, O.; Kennon, W.R.; Husain, M.D. Textile-based weft knitted strain sensors: Effect of fabric parameters on sensor properties. Sensors 2013, 13, 11114-11127. [CrossRef] [PubMed]

24. Metcalf, C.D.; Collie, S.; Cranny, A.; Hallett, G.; James, C.; Adams, J.; Chappell, P.; White, N.; Burridge, J. Fabric-based strain sensors for measuring movement in wearable telemonitoring applications. In Proceedings of the IET Conference on Assisted Living, London, UK, 24-25 March 2009.

25. Stewart, R.; Skach, S. Initial Investigations into Characterizing DIY E-Textile Stretch Sensors. In Proceedings of the 4th International Conference on Movement Computing, London, UK, 28-30 June 2017. 
26. Hallam, J.; Keen, E.; Lee, C.; McKenna, A.; Gupta, M. Ballet hero: Building a garment for memetic embodiment in dance learning. In Proceedings of the 2014 ACM International Symposium on Wearable Computers: Adjunct Program, Washington, DC, USA, 13-17 September 2014; pp. 49-54.

27. Karpashevich, P.; Hornecker, E.; Honauer, M.; Sanches, P. Reinterpreting Schlemmer's Triadic Ballet: Interactive Costume for Unthinkable Movements. In Proceedings of the $2018 \mathrm{CHI}$ Conference on Human Factors in Computing Systems, Montreal, QC, Canada, 21-26 April 2018; p. 61.

28. Stewart, R. Cords and Chords: Exploring the Role of E-Textiles in Computational Audio. Front. ICT 2019, 6, 2, doi:10.3389/fict.2019.00002. [CrossRef]

29. EeonTexTM LTT-SLPA. Eeontex Conductive Stretchable Fabric; Eeonyx Corporation: Pinole, CA, USA, 2009.

30. Hoffmann, K. Applying the Wheatstone Bridge Circuit; HBM: Darmstadt, Germany, 1974.

(C) 2019 by the authors. Licensee MDPI, Basel, Switzerland. This article is an open access article distributed under the terms and conditions of the Creative Commons Attribution (CC BY) license (http:// creativecommons.org/licenses/by/4.0/). 